\section{POLARIZED DEUTERON TARGET IN AN ELECTRON STORAGE RING: MEASUREMENTS AND PERSPECTIVES}

DE89 005890

S. I. Mishnev, D. M. Nikolenko, S. G. Popov, I. A. Rachek, A. B. Temnykh, D. K. Toporkov, E. P. Tsentalovich, D. K. Vesnovsky, B. B. Wojtsekhowski, V. G. Zelevinsky

Institute of Nuclear Physics, Novosibirsk, 630090, USSR

R. Gilman, R. J. Holt, E. R. Kinney, R. S. Kowalczyk, J. Napolitano, L. Young

Argonne National Laboratory, Argonne, IL 60439-4843

\begin{abstract}
A gas jet of polarized deuterium atoms with a thickness of $2 \times 10^{11}$ $\mathrm{cm}^{-2}$ has been used as an internal target in the VEPP-3 electron storage ring with $2 \mathrm{GeV}$ energy. Source characteristics as well as experimental conditions and results on elastic and inelastic scattering are reviewed.

Results of experimental studies and calculations of jet depolarization induced by an electron beam are discussed. For the first time a storage cell for polarized atoms was used in the storage ring in order to increase the target thickness. Depolarization of atoms inside the cell due to wall collisions with a drifilm surface and a magnetic field is proved to be small whereas depolarization due to the transitional magnetic field of the electron beam at $2 \mathrm{GeV}$ energy can be significant.

Conditions for small depolarization by the electron beam were found. An effective target polarization in the storage cell was found to be $\mathrm{P}_{\mathrm{zz}}^{\mathrm{eff}}=0.8 \pm 0.2$.
\end{abstract}

\title{
INTRODUCTION
}

Experiments with an internal target in a storage ring for charged particles have certain advantages as compared with using linear accelerators, as was shown by Budker et al. ${ }^{1}$

There are specific advantages of a storage ring operation with an internal target:

i) nearly CW operation;

ii) high luminosity at high experimental accuracy;

iii) various possibilities of detecting secondary particles (including very low energy particles) due to the small target thickness;

iv) possibility of using special targets (for example gas polarized jets) and exotic stored beams (positrons, antiprotons, polarized electrons). 
The polarized gas jet target was designed and fabricated in Novosibirsk to investigate properties of a deuteron, the simplest nuclear system. $^{2}$ Electron scattering by polarized deuterons is a good check for various theoretical predictions.

There are three experimental approaches to solve this problem:

i) unpolarized target and measurement of tensor polarization of the recoil deuteron; ${ }^{3}$

ii) tensor polarized deuteron target of solid $\mathrm{ND}_{3}$ for elastic electron scattering; ${ }^{4}$

iii) internal polarized gas jet target in an electron storage ring and asymmetry measurement in elastic and inelastic electron scattering. ${ }^{5}$

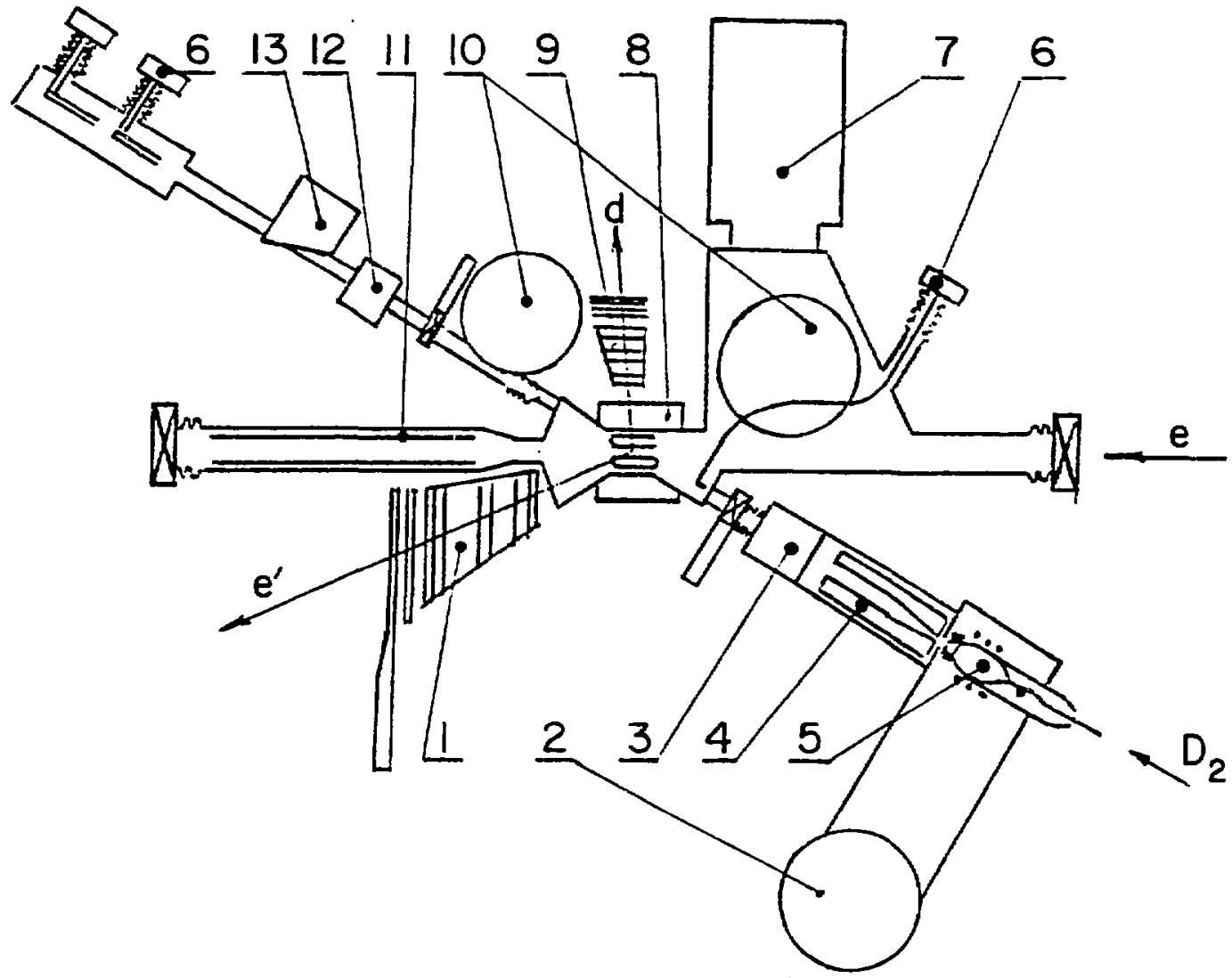

Fig. 1. Schematic view of the experimental set up: 1-detector system for scattered electrons; 2-cryopump; 3-HF transition unit (HF1) 4-sextupole magnet; 5-discharge tube; 6-movable vacuum gauge; 7-ion pump; 8-magnet for holding field; 9-detection system for heavy particles; $10-T_{i}$ sublimation pump; 11-ion trap; 12-HF transition unit (HF2); 13-Rabi magnet. 
Each method mentioned above has its shortcomings: the first one demands a polarimeter of high efficiency and wide energy range; in the second case there is a problem of polarization stability against radiation damage; in the last case the main problem is to produce a polarized target of high density.

In this report we present the first results for a polarized deuterium jet target in the VEPP-3 electron storage ring. In addition, we report on the first use of a storage cell for polarized atoms in an electron ring environment. This latter work is the result of a collaborative effort between Argonne National Laboratory and the Institute of Nuclear Physics at Novosibirsk.

\section{EXPERIMENT AT THE VEPP-3 ELECTRON STORAGE RING}

Figure 1 gives a general view of the experimental equipment installed in the second straight section of the storage ring. The experiment was performed at an energy of $2 \mathrm{GeV}$ and electron current of $0.2-0.1 \mathrm{~A}$, the larger value being close to the limiting current in the storage ring. Four identical detection systems, placed symmetrically with respect to the electron beam axis, were used for detection of scattered electrons throughout the angular range $\theta_{\mathrm{e}}=10^{\circ}-20^{\circ}$ and $\phi_{\mathrm{e}}= \pm 20^{\circ}$, and for registration of heavy particles with emission angles $\theta_{\rho}=70^{\circ}-80^{\circ}$ and $\phi_{\rho}= \pm 20^{\circ}$. The polarized atomic beam source was installed in the median plane of the storage ring. To control the polarization direction in the intersection region of the gas get with the electron beam, a 0.5 kG magnetic field was changed in 2-minute intervals along directions $\phi_{\pi}=0$ and $\phi_{\pi}=90^{\circ}$, perpendicular to the electron beam axis. The gas pressure in the straight section was $P_{\text {exp }} \sim 10^{-7}$ Torr during the experiment and $P_{b} \leq 10^{-8}$ Torr without a gas jet. This pressure does not influence the lifetime $\tau \simeq 10^{4} \mathrm{~s}$ of the electron beam in the ring. The amount of charge crossing the jet in a 12 hour period was typically 5 $\mathrm{kC}$. Figure 2 illustrates typical time dependence of the electron current in the electron storage ring.

\section{ATOMIC BEAM SOURCE}

The source of polarized deuterium atoms, made according to the classical scheme, produces the total flux of polarized atoms of $2 \times 10^{16}$ atoms $/ \mathrm{s}$ and gives a jet target thickness ${ }^{2}$ of $2 \times 10^{11}$ atoms $/ \mathrm{cm}^{2}$. In order to achieve a maximum density, the behavior of molecular beam intensity versus the flow through the nozzle and versus the nozzleskimmer distance was studied.

\section{DISCLAIMER}

This report was prepared as an account of work sponsored by an agency of the United States Government. Neither the United States Government nor any agency thereof, nor any of their employees. makes any warranty, express or implied, or assumes any legal liability or responsibility for the accuracy, completeness. or usefulness of any information, apparatus, product. or process disclosed, or represents that its use would not infringe privately owned rights. Reference hereın to any specific commercial product. process, or service by trade name. trademark. manufacturer. or otherwise does not necessarily constitute or umply its endorsenent, recommendation, or favoring hy the United States Government or any agency thereof The views and opinions of authors expressed herein do not necessarily state or reflect those of the United States Government or any agency thereof. 


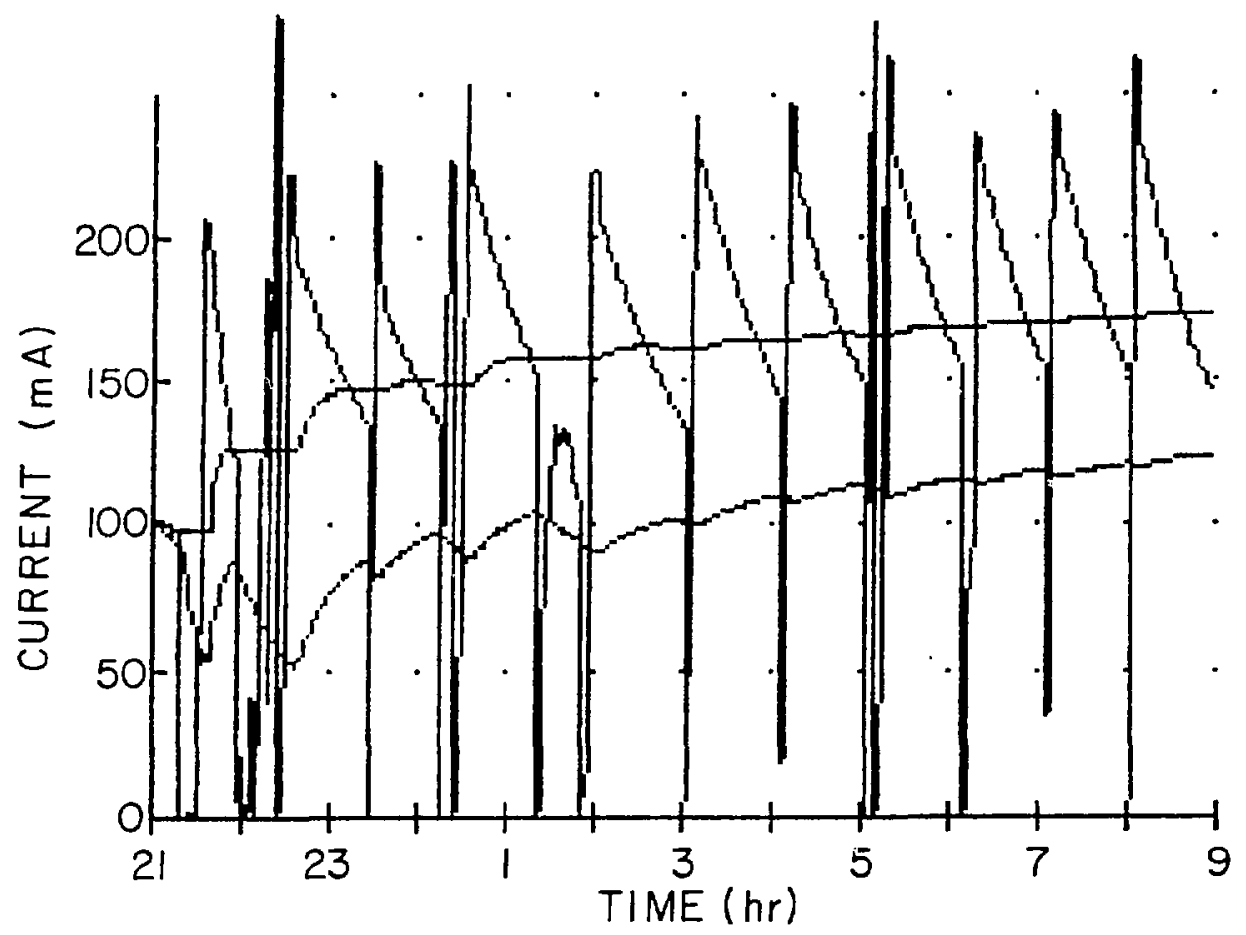

CHARGE $=5317.9$ Coul.

Fig. 2. Time dependence of the electron current in the electron storage ring during experiment with an internal target.

The nozzle made from aluminum has a 2-mm diameter channel. Atomic deuterium, passing through the liquid-nitrogen cooling nozzle, is pumped by a $1500 \mathrm{\ell} / \mathrm{s}$ cryopump. The sextupole magnet with a length of $30 \mathrm{~cm}$ is installed after the skimmer. Its aperture was designed to obtain maximum intensity of the focussed atomic beam. The aperture varies from $8 \mathrm{~mm}$ at the entrance of the magnet to $25 \mathrm{~mm}$ at the center of the sextupole and remains constant at $25 \mathrm{~mm}$ to the exit. A superconducting sextupole is used in order to achieve high pole tip field and its cover acts as a cryopump. Figure 5 (curve 1) shows the intensity distribution of the focussed atomic beam measured in the vacuum chamber of the storage ring. The full width of the beam is 1 $\mathrm{cm}$. The dissociation degree in the quartz discharge tube exceeds $50 \%$ and does not change significantly during a two week period. After two weeks of operation it was necessary to remove deuterium from the source and clean the tube with hydrofluoric acid, distilled water and a weak solution of boric acid. The nozzle was cleaned by orthophosphoric acid and distilled water. 


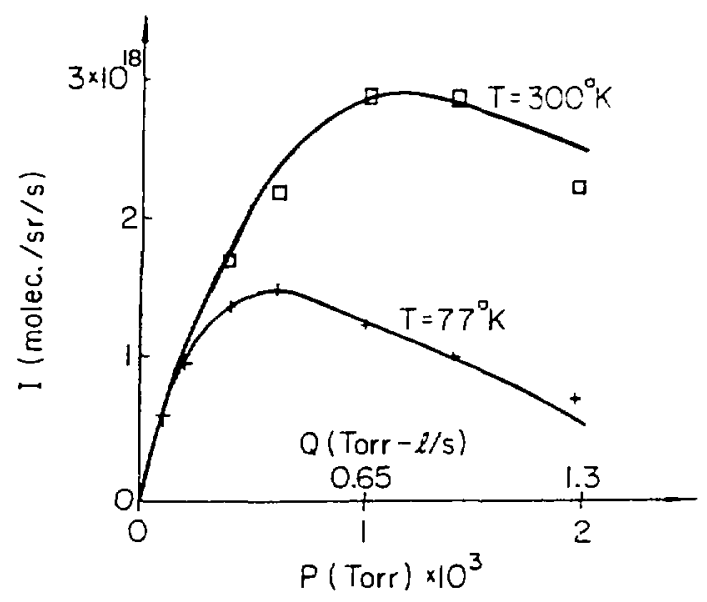

Fig. 3. Intensity of the molecular deuterium beam as a function of the gas flow and pressure in the nozzle chamber for two temperatures of the nozzle (solid lines denote the calculation, reference for curves; square and + denote the measured quantities).

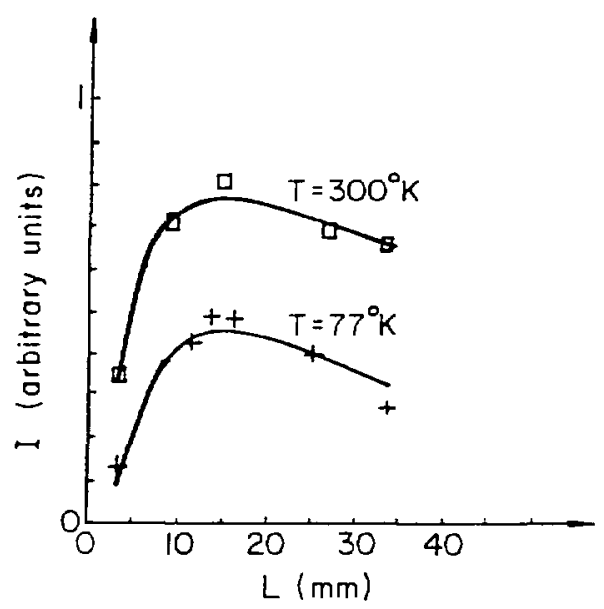

Fig. 4. Intensity of the beam of molecular deuterium vs the nozzle-to-skimmer distance at the gas flow $Q=0.4$ Torr $/ \mathrm{s}$ for two temperatures of the nozzle (solid lines stand for the calculation, reference for curves; + and square denote the measured quantities). 


\section{MEASUREMENT OF ATOMIC BEAM POLARIZATION}

The measurement of atomic beam polarization was based on the deflection of atoms by the non-uniform magnetic field of the Rabi magnet. The deflection angle is

$$
\delta=\mu \mathrm{B} /\left(\mathbf{m} \mathrm{v}^{2} \mathrm{~g}\right),
$$

where $\mu$ is the magnetic moment projection of the atom along the magnetic field $B, m$ and $v$ are the mass and the velocity of the atom respectively, and $\theta$ is the angle between the velocity direction and the direction of the pole-piece of the Rabi magnet. Before the Rabi magnet there is a screen with a slit of $1 \times 10 \mathrm{~mm}$ for forming the narrow atomic beam. Figure 5 (curve 2) shows the space distribution of the intensity of the atomic beam after the Rabi magnet when the magnet is turned off. If the magnet is turned on the beam moves as a whole to the right side (curve 3). After the HF transition, a part of the atomic beam is deflected by the magnet to the other size (curve 4).

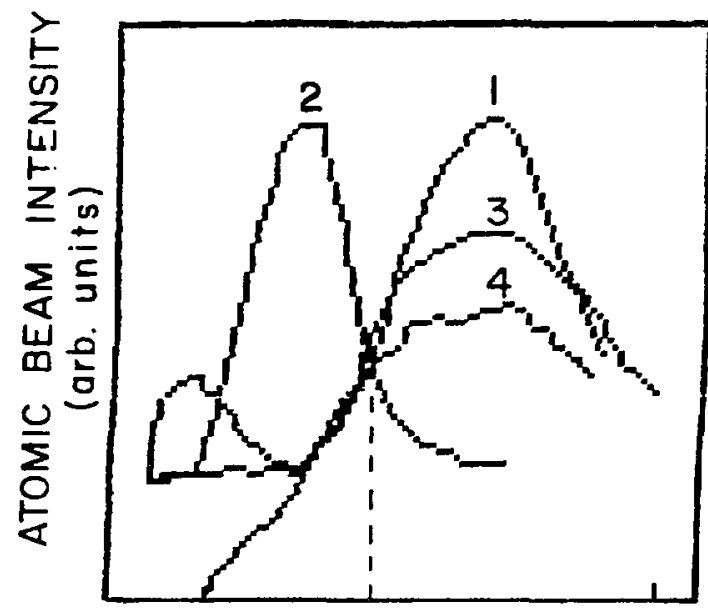

Fig. 5. Space beam profile: 1-measured in the vacuum chamber of the storage ring, FWHM of the beam $1 \mathrm{~cm}$; 2,3,4-measured behind the Rabi magnet: 2-Rabi magnet off; 3-magnet on; 4-magnet on/off transition on.

The actual tensor polarization of the atomic beam is determined by the efficiency of $\mathrm{HF}$ transitions

$$
P_{z Z}= \pm 3\left(1-A_{4} / A_{3}\right) \text {, }
$$

where $A_{3}$ and $A_{4}$ are average intensities shown in the right part of curves 3 and 4 (Fig. 5). The sign \pm in Eq. (2) corresponds to the HF transition $2^{++6}\left(3^{++5}\right)$. Figure 6 shows the distribution of $P_{z z}$ measured under experimental conditions during two months. 


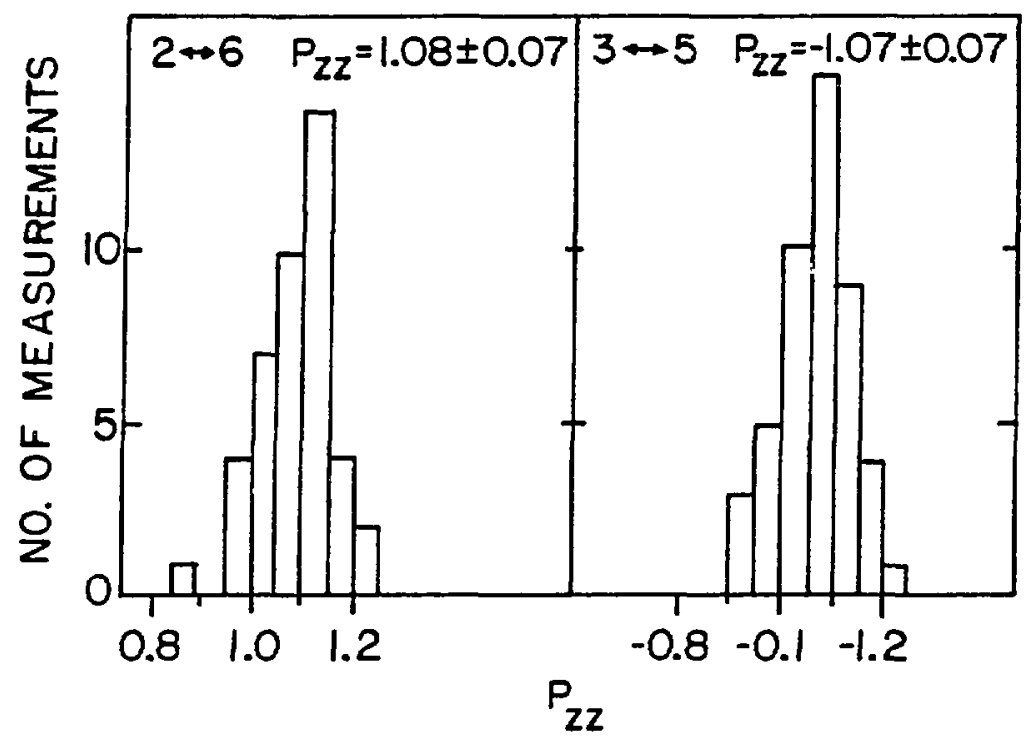

Fig. 6. The distribution of $P_{z z}$ measured under experimental conditions.

The average value $P_{2 x}$ differs from unity. This can be explained by absorption and dynamic flow processes in the vacuum gauge. For polarization control the movable channel was set at the position corresponding to the maximum intensity of atoms under going the HF transition. The difference of signals was measured for the Rabi magnet on and off. The effective deuteron polarization $P_{z 2}^{\text {eff }}$ in the intersection region of the gas jet when the electron beam is less than $P_{z z}$. This reduction is due to the finite value of the holding magnetic field, depolarizating influence of the electron beam, confinement of unpolarized ions near the electron beam and the unpolarized target background. The effective polarization is $\mathrm{P}_{\mathrm{zz}}^{\mathrm{eff}}=0.65$.

EXPERIMENTAL RESULTS FOR ELASTIC AND INELASTIC ed SCATTERING USING A DIRECT JET AS A TARGET The counting rate of the reaction under study $R_{H, S}^{N}$ was measured, where $\mathrm{N}=1,2,3,4: \mathrm{H}=1,2: \mathrm{S}=1,2$ stand for the number of the detection system, the direction of the governing field and the type of $\mathrm{HF}$ transition respectively. For electrodisintegration reaction, the asymmetry was determined (Fig. 7) and for elastic scattering the component of the analyzing power $\mathrm{T}_{20}$ was extracted (Fig. 8). 


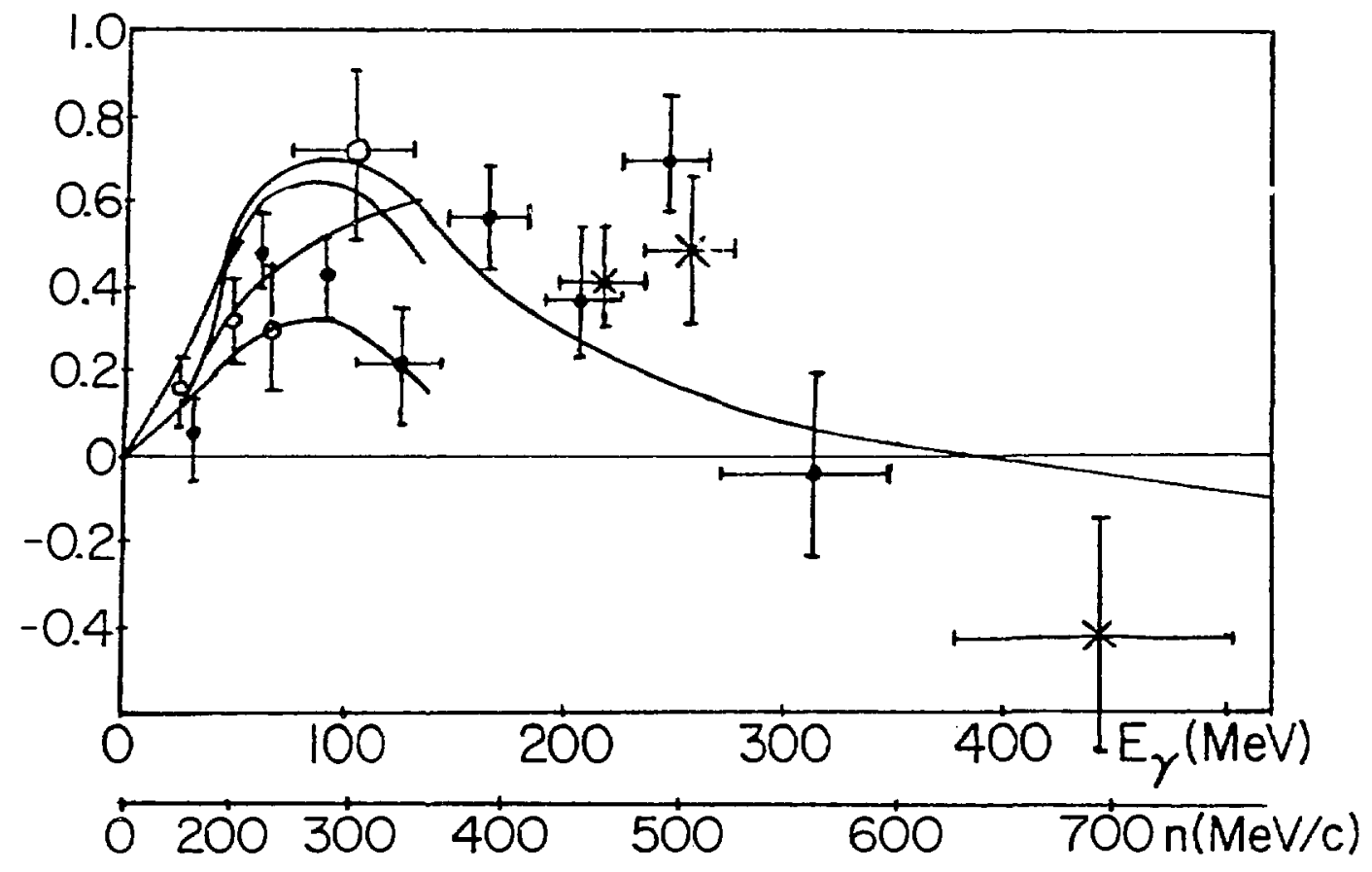

Fig. 7. The asymmetry of the đ(e,pn)e' reaction: 1-PWA calculation with the Hamada-Johnston potential; 2-the same as 4 for the Reid soft-core potential; ${ }^{7}$ 3-relativistic impulse approximation; ${ }^{8}$ 4-nonrelativistic calculation with a final state interaction for the Paris potential. Open circles-VEPP-2 (Ref. 14); darkened circles-VEPP-3; x-VEPP-3 with electron tagging.

The error bars shown in Fig. 7 are only of statistical origin. For improved accuracy and measurements at higher momentum transfer, it is necessary to use a thinner target.

\section{THE STORAGE CELL}

The possibility to store polarized $H$ atoms in a cell was demonstrated in Ref. 15. In Ref. 16 a storage cell has been proposed for use as an internal gas target in a storage ring. The density $n$ of atoms in the cell can be defined from the flow of atoms into the cell and the conductance $\mathrm{C}$ of its channels

$$
\mathrm{n}_{\mathrm{c}}=\mathrm{Q} / \mathrm{C} \text {. }
$$




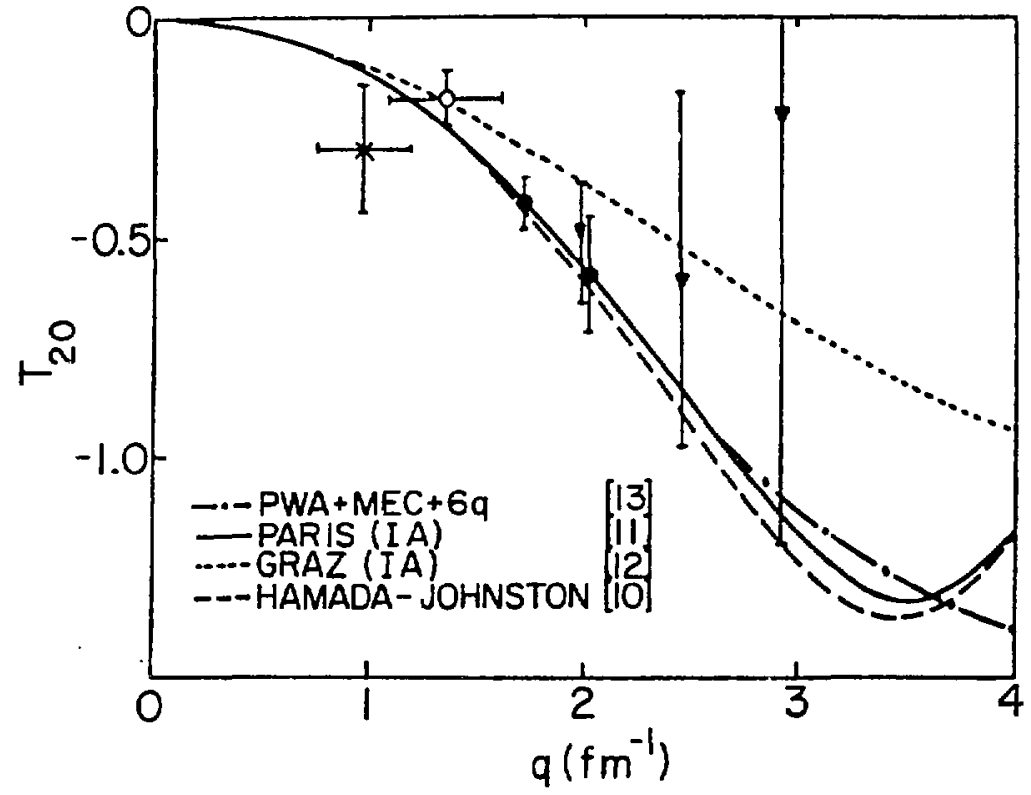

Fig. 8. Tensor analyzing power $T_{20}$. Solid points-data of Ref. 3; x-Ref. 9; 0-Ref. 5; $\Delta$-present work with polarized jet target.

For channels consisting of tubes with the diameter $d_{i}$ and the length $\ell_{i}$

$$
\mathrm{C}=2.8 \times 10^{3} \sqrt{\mathrm{T} / \mathrm{M}} \sum \mathrm{d}_{i}^{2} /\left[1+\frac{3 \ell_{i}}{4 \mathrm{~d}_{i}}\right) \mathrm{cm}^{3} / \mathrm{s}
$$

where $T$ and $M$ are temperature and atomic mass respectively. The average dwell time $\tau$ of atoms in the cell is $\tau=\mathrm{V} / \mathrm{C}$, where $\mathrm{V}$ is the cell volume. Before escape, an atom undergoes the number of wall collisions $\nu$,

$$
\nu=v_{c} \times \tau / \lambda .
$$

Here $v_{c}=1.45 \times 10^{4} \sqrt{\mathrm{T}} 7 \overline{\mathrm{M}} \mathrm{cm} / \mathrm{s}$ is an average velocity of an atom in the cell, $\lambda$ characterizes the mean path between collisions with the wall. The atomic density is given in the $T$-shape storage cell with equal arms and $L / d>1$ by

$$
n_{c}=n_{b} \frac{L v_{b}}{d v_{c}},
$$

where $v_{b}$ is a velocity of atoms in the beam. 
The central concerns for studying depolarization of atoms in the storage cell were the effects of the high frequency magnetic field of the electron bundles and collisions of atoms with the cell wall. The effective polarization of the target is reduced by less than $10 \%$ due to trapping of deuterons by the electron beam.

\section{DEPOLARIZATION OF ATOMS DUE TO THE PERIODIC} HIGH FREQUENCY OF THE ELECTRON BUNCH

Experimental measurements and numerical calculations have been carried out to study depolarization due to the magnetic field of the electron bunch. With the help of high frequency unit (HF1) the atomic beam has been prepared in one of three states

$$
\left(w_{1}, w_{2}, w_{3}, w_{4}, w_{5}, w_{6}\right)=\begin{aligned}
& (1 / 3,1 / 3,1 / 3,0,0,0) \\
& (1 / 3,0,1 / 3,0,0,1 / 3) \\
& (1 / 3,1 / 3,0,0,1 / 3,0)
\end{aligned} .
$$

where $W_{m}$ is the population of the magnetic substate $m$. After the atomic beam crosses the electron bunch, the final state was analyzed by the high frequency unit (HF2) and the Rabi magnetic (see Fig. 1). In this case, the detector was positioned so that only atoms with $m_{z}=-1 / 2$ were detected.

$$
\begin{aligned}
w_{4}^{+}+w_{5}^{+}+w_{6}^{+} & \text {HF2 }=0 \\
\Lambda=w_{4}^{+}+w_{5}^{+}+w_{2}^{+} & \text {HF2 }=[2++6] \\
w_{4}^{+}+w_{3}^{+}+w_{6}^{+} & \text {HF2 }=[3++5]
\end{aligned}
$$

where $\mathrm{W}_{\mathrm{m}}^{+}$is the population of the substate $\mathrm{m}$ after crossing the beam by the electron bunch.

The computation program solves the time-dependent Schrödinger equation

$$
i \hbar \frac{\partial \Psi}{\partial t}=B \Psi
$$

where

$$
\begin{aligned}
& \mathrm{H}=\mathrm{H}_{0}+\mathrm{V}, \mathrm{H}_{0}=\mathrm{a} \neq \cdot \vec{J}+\left[\frac{\mu_{\mathrm{J}}}{\mathrm{J}_{\mathrm{c}}} \mathrm{J}+\frac{\mu_{\mathrm{I}}}{\mathrm{I}}\right] \cdot \cdot \mathrm{B}_{0}, \\
& \mathrm{~V}=\left[\frac{\mu_{\mathrm{J}}}{\mathrm{J}} \mathrm{J}+\frac{\mu_{\mathrm{I}}}{\mathrm{I}} \mathrm{t}_{]}\right] \cdot \overrightarrow{\mathrm{B}}_{\mathrm{v}}(\mathrm{t}) .
\end{aligned}
$$

where $\vec{B}_{0}$ is the holding static magnetic field and $\vec{B}_{v}(t)$ is the timevarying magnetic field of the electron bunch. The space-time density 
distribution of the electron current is assumed to be of Gaussian form:

$$
i(t, \rho)=I_{0} T / \tau \frac{1}{\sqrt{2 \pi}} \exp \left(1-(t-n T)^{2} / 2 \tau^{2}\right) \frac{1}{\sqrt{2 \pi r_{b}}} \exp \left(-\rho^{2} / 2 r_{b}^{2}\right)
$$

where $I_{0}$ is the overage electron current, $T=250 \mathrm{~ns}$ is the period of beam circulation, $\tau=0.5 \mathrm{~ns}$ is the beam duration, and $\mathrm{r}_{\mathrm{b}}$ is the beam radius. The solution is of the form

$$
\begin{aligned}
& \Psi=\sum_{a=1}^{6} c_{n} \exp \left(-i E_{n} t / \hbar\right)|n\rangle, \quad n=\left|m_{J} I\right\rangle \\
& i \hbar C_{n}=E_{n} C_{n}+\sum_{i=1}^{6} C_{j} V_{n j} \exp \left(-i\left(E_{j}-E_{n}\right) / \hbar\right)
\end{aligned}
$$

Figure 9 shows measured and calculated depolarization effects as dependent dependent on the electron current in the storage ring. In the case when the holding magnetic field $B>>B_{c}\left(B_{c}=117 \mathrm{G}\right)$, the transition probabilities changing the nuclear angular momentum projection are small since $P_{n j} \sim\left|V_{n j}\right|^{2} \sim\left(B_{c} / B_{0}\right)^{2}$. However, resonant transitions are possible, because the influence of the electron beam is periodic and atoms are moving slowly enough (time-of-flight through the target region corresponds to 400 cycles of the electron bunch). Figure 10 shows the transition energy and resonance positions. The amplitude of $k$-th harmonic of the circulation frequency of the electron beam

$$
a_{x}=2 I_{0} \exp \left(-2(\pi k \tau / T)^{2}\right)=2 I_{0} \exp \left(-(k / 112.5)^{2}\right)
$$

the resonance width for the $n \rightarrow j$ transition is $\epsilon \simeq 4\left|V_{j n} / \hbar\right|$. Table I gives the widths of resonances and their positions for transitions shown in Fig. 10 at $f_{\text {circ }}=4030 \mathrm{kHz}$ and $\mathrm{I}_{0}=0.2 \mathrm{~A}$, typical for the VEPP-3 storage ring. The use of the holding magnetic field far from the resonances is preferable. The holding field $B_{0}=1400 \mathrm{G}$ was chosen for the new experimental design.

Table I

\begin{tabular}{ccccccccc}
\hline $\mathrm{B}_{0}(\mathrm{G})$ & 567.0 & 649.0 & 749.2 & 988.6 & 1116.5 & 1865 & 1885 & 1974 \\
\hline $\mathrm{k}$ & 31 & 24 & 30 & 25 & 29 & 27 & 27 & 26 \\
Trans. & $4-5$ & $1-2$ & $4-5$ & $1-2$ & $4-5$ & $2-3$ & $5-6$ & $1-2$ \\
$\epsilon(\mathrm{G})$ & 3.1 & 4.0 & 4.2 & 6.0 & 6.3 & 13.6 & 13.0 & 12.0 \\
\hline
\end{tabular}



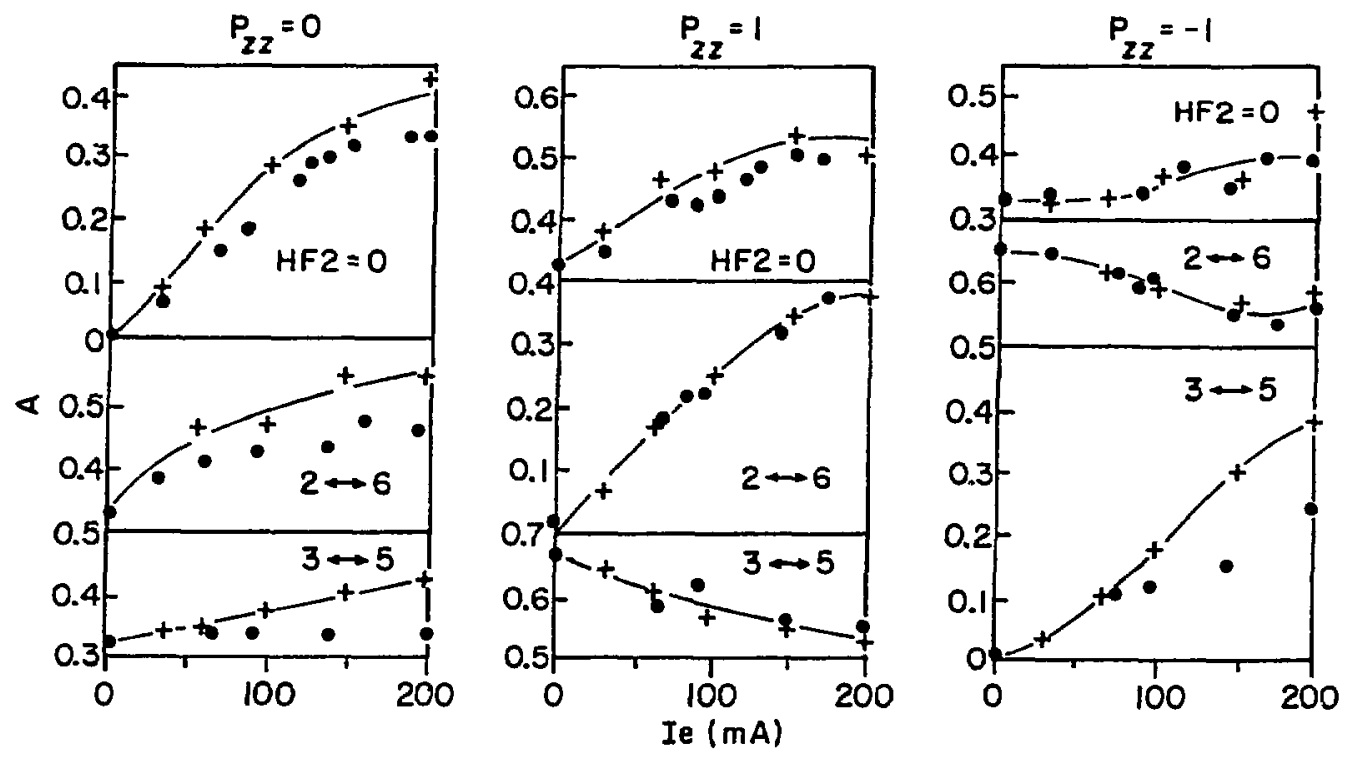

Fig. 9. Dependence of the depolarization of the atomic beam on the electron current in the storage ring (electron energy $2.0 \mathrm{GeV}$, magnetic guide field $130 \mathrm{G}, .0$ - measurements, +++ -calculations). Curves are for a guide to the eye only.

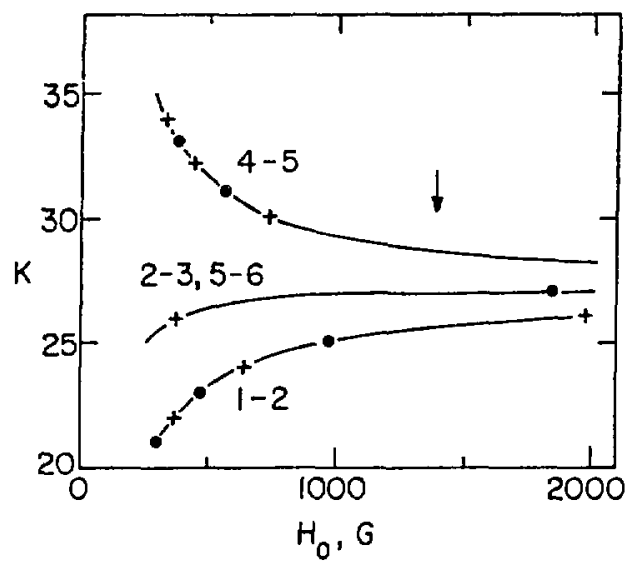

Fig. 10. Transition energy and resonance positions: $\left.\mathrm{k}=\left(\Delta \mathrm{E}_{\mathrm{n}} / \hbar\right) / \mathrm{f}_{\text {circ }}, \mathrm{f}_{\text {circ }}=4090 \mathrm{kHz}\right)$. For two bunches of the same value there are only even number resonances. + denotes even and dark circle represents odd resonances. 
Using several electron bunches in the ring is desirable to decrease depolarization effects. For the same average electron current it, would reduce the number of resonant points (Fig. 10) and decrease the transition probability, since for $m$ electron bunches

$$
P_{m} \sim m\left|V_{m}\right|^{2} \simeq m\left|\frac{v_{1}}{m}\right|^{2}=\left|\frac{v_{1}}{m}\right|^{2} .
$$

Presently, it is feasible to use two electron bunches in the VEPP-3 storage ring.

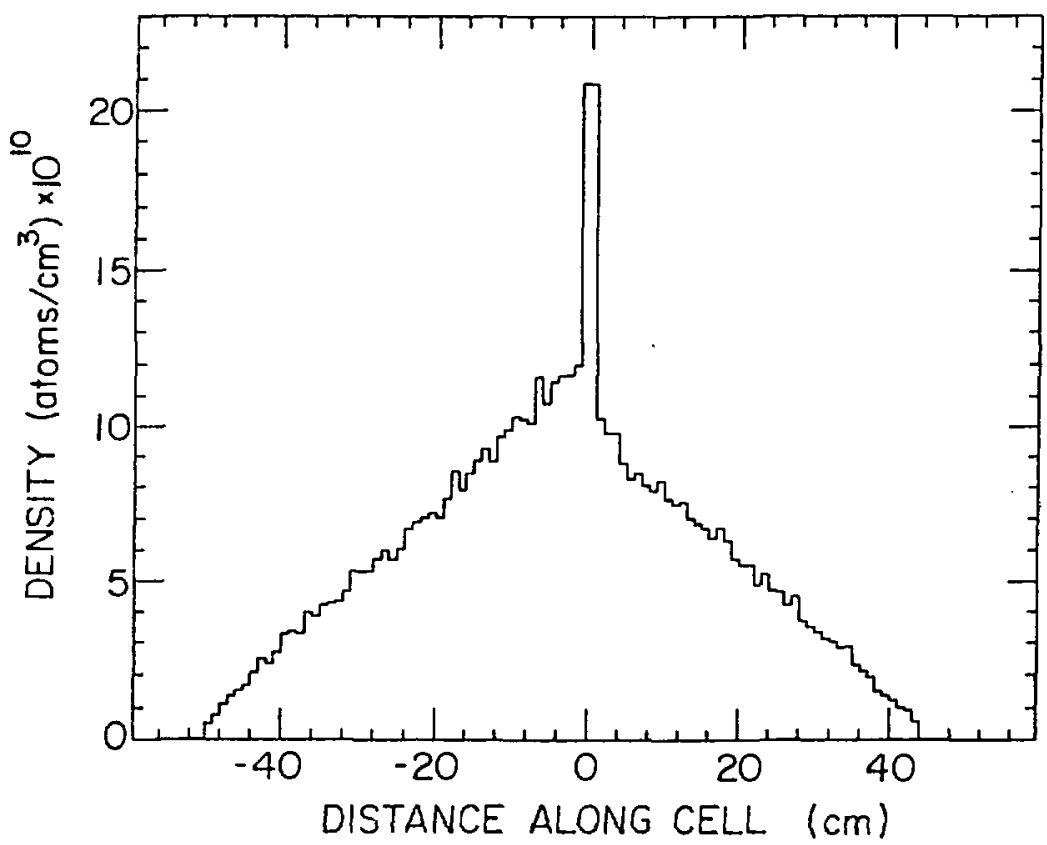

Fig. 11. The density distribution of atoms in the cell determined by a Monte Carlo analysis.

\section{DEPOLARIZATION OF ATOMS BY WALL COLLISIONS IN THE STORAGE CELL}

To improve the density and target thickness, an aluminum storage cell fabricated at Argonne was installed into the vacuum chamber of the storage ring. Its length is $940 \mathrm{~mm}$, cross section has an elliptic shape $24 \times 46 \mathrm{~mm}$ being determined by the electron beam aperture during injection. Polarized atoms were injected into the cell through a channel of $60 \mathrm{~mm}$ length and $10 \mathrm{~mm}$ diameter. The walls of the cell were coated with "drifilm" having a large spin-relaxation time. ${ }^{17}$ Figure 11 shows the density distribution of the atoms in the cell found by Monte Carlo simulation. It is assumed that the flow of atoms into the cell is 
$10^{16}$ atoms $/ \mathrm{s}$, the velocity of entering and reflecting atoms are $\mathrm{V}_{\mathrm{an}}=10^{5}$ $\mathrm{cm} / \mathrm{s}, \mathrm{r}_{\mathrm{ref}}=1.9 \times 10^{5} \mathrm{~cm} / \mathrm{s}$ respectively. The average number of wall collisions is $\mathrm{v}=470$ and average dwell time in the cell is $5.6 \mathrm{~ms}$.

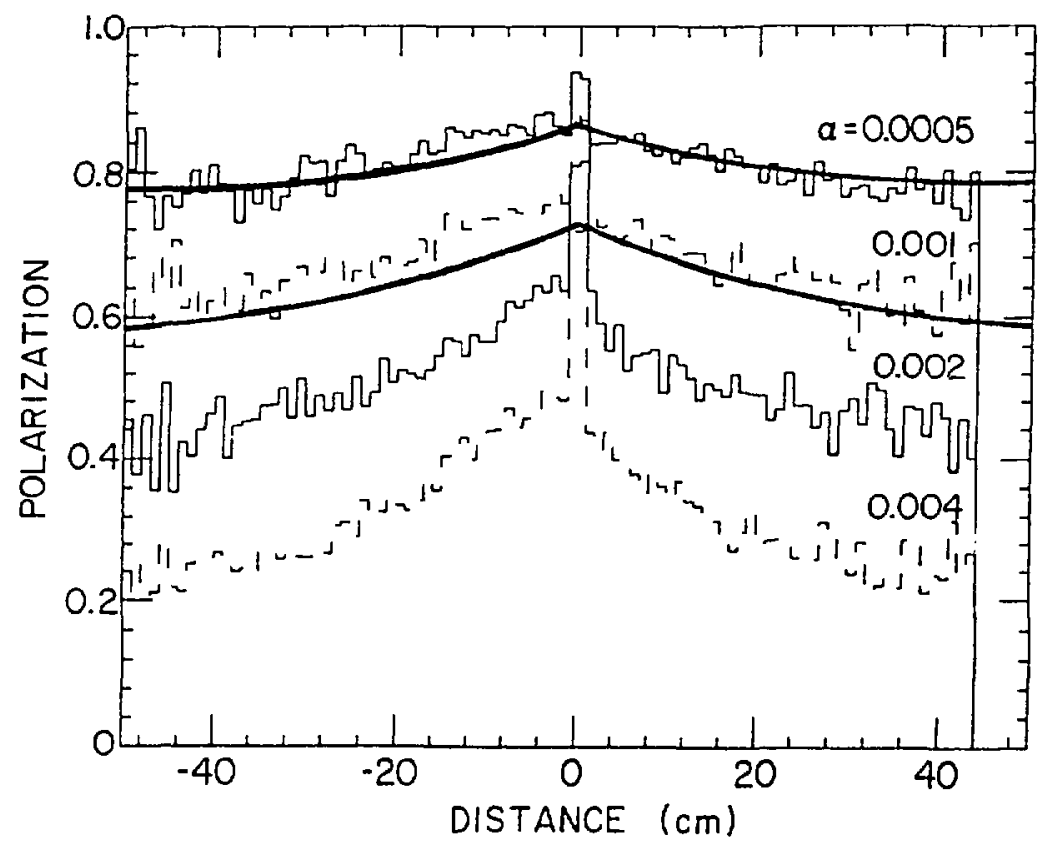

Fig. 12. Results of a Monte Carlo anclysis of the polarization distribution in the cell (solid curves-analytical estimates). The quantity $a$ is the depolarization probability per cell wall collision.

Figure 12 shows the polarization distribution along the cell for several values of depolarization probability per cell-wall collision. The solid curve is an analytical estimate of depolarization, assuming that depolarization is small and density distribution is linear in the cell.

\section{EXPERIMENTAL RESULTS WITH THE STORAGE CELL IN THE ELECTRON STORAGE RING}

The use of the storage cell increased the counting rate by a factor of 3 compared with the jet target. Figure 13 shows coordinate distributions of events along the electron beam for three sets of data: i) using the direct jet as a target; ii) under $D_{2}$ vacuum conditions; and iii) using the storage cell. 


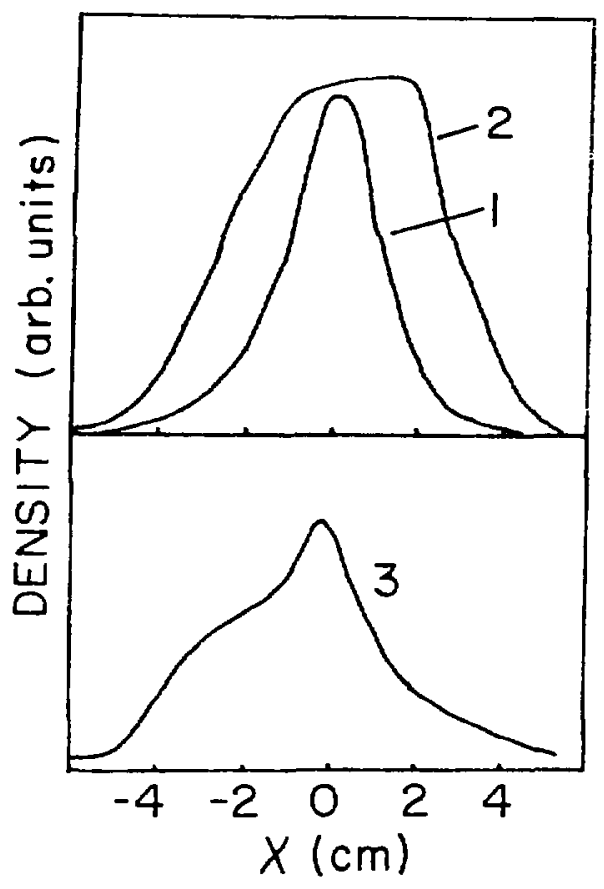

Fig. 13. Coordinate distributions of events along the electron beam: 1 -jet; 2- $\mathrm{D}_{2}$ vacuum; 3 -storage cell.

Curve 2 in Fig. 13 indicates the detector acceptance since the target area is filled with $\mathrm{D}_{2}$ gas with storage cell in place. Curve 3 strongly resembles the expected distribution of Fig. 11 if the detector acceptance and the loss of low energy protons is taken into account. For decrease of depolarization due to the electron beam, a magnetic field was applied.

This field cannot be produced uniformly along the whole cell due to construction of the straight section and the detection system. Table II shows the results of the asymmetry measurements of the reaction d $(e, p n) e^{\prime}$. At the electron energy $0.6 \mathrm{GeV}$ when the beam length is $\tau=4 \mathrm{~ns}$ and the depolarization due to the electron beam is small, the asymmetry measured using the storage ell is consistent with that using the direct jet. This demonstration within the error bars, that depolarization due to the wall, vollisions are absent. At the electron energy $2.0 \mathrm{GeV}$, where the pulse width is $0.5 \mathrm{~ns}$, the measured asymmetry is close to zero and indicates strong depolarization by the electron beam. This can be explained by resonant depolarization induced by the non-uniform magnetic field. The final entry in the table was taken with the short pulse, but the holding magnetic field was increased to $350 \mathrm{G}$ at minimum rather than $250 \mathrm{G}$. Here the polarization of the target is preserved.

Table II

\begin{tabular}{lclll}
\hline Type & $\mathrm{E}(\mathrm{GeV})$ & $\mathrm{N}$ bunch $\mathrm{I}(\mathrm{mA})$ & $\tau(\mathrm{ns})$ & asymmetry \\
\hline Jet & 2 & $1 \times(200-100)$ & 0.5 & $0.235 \pm 0.026$ \\
Cell & 2 & $1 \times(200-100)$ & 0.5 & $0.02 \pm 0.03$ \\
Cell & 0.6 & $1 \times(100-100)$ & 4 & $0.23 \pm 0.06$ \\
Cell & 2 & $2 \times(140-70)$ & 0.5 & $0.28 \pm 0.06$ \\
\hline
\end{tabular}




\section{CONCLUSIONS}

We have demonstrated the first use of a storage cell for polarized deuterium atoms in an electron storage ring. The depolarization of atoms induced by wall collisions on a drifilm surface in a magnetic field was found to be small. The depolarization of atoms due to the magnetic field of an electron beam can be significant. The experimental results of depolarization of atoms by the electron beam are in good agreement with calculations. These results provide a basis for a new design of a storage cell which can increase the density by an order of magnitude using the existing source and increase the target thickness up to $\sim 5 \times 10^{12}$ atoms $/ \mathrm{cm}^{2}$.

This work supported in part by the U. S. Department of Energy, Nuclear Physics Division, under contract W-31-109-ENG-38.

\section{REFERENCES}

1. C. J. Budker et al., Yad. Fiz., Vol. 6, No. 4, 775 (1967).

2. A. V. Evstigneev, S. C. Popov and D. K. Toporkov, NIM A238, 12 (1985).

3. M. E. Shulze et al., Phys. Rev. Lett. 52, 597 (1984).

4. W. Mayer, Helvetica Phys. Acta, Vol. 59, No. 4, 728 (1986).

5. B. B. Wojtsekhowski et al., JETF Lett., Vol. $\underline{43}$, No. 12, 567 (1986).

6. M. V. Mostovoy, private communication.

7. H. Arenhövel, private communication.

8. M. P. Rekalo, G. I. Gakh and A. P. Rekalo, Phys. Lett. 166B, 27 (1986).

9. V. F. Dmitriev et al., Phys. Lett. 157B, 143 (1985).

10. T. Hamada and J. D. Johnston, Nucl. Phys. 34, 382 (1962).

11. M. Lacombe et al., Phys. Rev. C 21, 861 (1980).

12. L. Crepinsek et al., Acta Phys. Austr. 42, 139 (1975).

13. M. Beyer and H. J. Weber, Phys. Rev. C 35, 14 (1987).

14. M. V. Mostovoy et al., Phys. Lett. 188B, 181 (1987).

15. W. Haeberli et al., AIP Conference Proceedings 69, 931 (1980).

16. R. Holt, in Proceedings of the Spectrometers Workshop at the College of William and Mary, Oct. 10-23, 1983; Proceedings of the Workshop on Internal Targets in Storage Rings, Argonne ANL Report No. 84-50, 103 (1984); Proceedings of the International Conference on Interesections of High Energy and Nuclear Physics, Steamboat Springs, AIP Conference Proceedings 123, 499 (1984).

17. G. E. Thomas et al., NIM A257, 32 (1987); L. Young et al., Nucl. Instr. and Meth. B24/25, 963 (1987). 